\title{
NOUVELLe
}

\section{Une imagerie métabolique des cancers primitifs du foie fondée sur leur addiction à la choline prédit leur oncogénotype et ouvre une piste thérapeutique}

\author{
Angélique Gougelet, Sabine Colnot
}

Vers une imagerie métabolique des tumeurs cancéreuses

L'imagerie des tumeurs malignes par tomographie par émission de positons (TEP) utilise une propriété essentielle des cellules cancéreuses: leur métabolisme modifié afin d'acquérir une autonomie énergétique. Suite à l'injection d'un métabolite radio-marqué, la tumeur primaire et ses métastases éventuelles sont visualisées par l'incorporation accrue de ce métabolite dans les celIules tumorales par rapport aux cellules saines adjacentes [1] $(\rightarrow)$.

$(\rightarrow)$ Voir le Forum de J.L. Coatrieux et al., $\mathrm{m} / \mathrm{s} \mathrm{n}^{\circ} 12$, décembre 2010, page 1103

L'utilisation de cette technique d'imagerie dans le cancer a fait suite aux travaux réalisés par Otto Warburg dans les années 1930, qui avait montré «l'addiction» des tumeurs au glucose qu'elles utilisent comme substrat énergétique (Figure 1). La TEP a ainsi permis de visualiser bon nombre de tumeurs par leur addiction au fluorodésoxyglucose radio-marqué au fluor ${ }^{18} \mathrm{~F}$ (FDG). Les dernières décennies ont vu s'affiner cette notion d'addiction métabolique, ce qui a conduit à proposer divers substrats énergétiques pouvant être utilisés pour l'imagerie, traduisant la diversité métabolique des tumeurs cancéreuses [2] $(\rightarrow)$.

$\rightarrow$ Voir la Synthèse de M. Cordier-Bussat et al., $m / s n^{\circ} 8-9$, août-septembre 2018, page 701

C'est ainsi qu'une nouvelle imagerie métabolique s'est récemment dévelop-
Équipe «Rôles oncogéniques de la signalisation par la $\beta$-caténine dans le foie », labellisée par la Ligue nationale contre le cancer, Centre de recherche des Cordeliers, Inserm, Sorbonne Université, Université de Paris, UMRS1138, 15 rue de l'École de médecine, 75006 Paris, France.

angelique.gougelet@inserm.fr sabine.colnot@inserm.fr

pée. Elle permet certes de diagnostiquer la présence de tumeurs, mais le choix du traceur radioactif vise maintenant à les stratifier, afin de proposer au patient un traitement personnalisé adapté au tissu d'origine de la tumeur, ainsi qu'à ses caractéristiques métaboliques, morphologiques et génétiques.

Le cas du carcinome hépatocellulaire Le carcinome hépatocellulaire ( $\mathrm{CHC}$ ) est le cancer primitif du foie le plus fréquent, survenant le plus souvent dans un foie cirrhotique associé à une hépatite chronique sous-jacente liée aux infections par les virus de l'hépatite B ou C, à l'alcoolisme, ou à l'obésité et au diabète. Dans le CHC, un certain nombre de mutations 


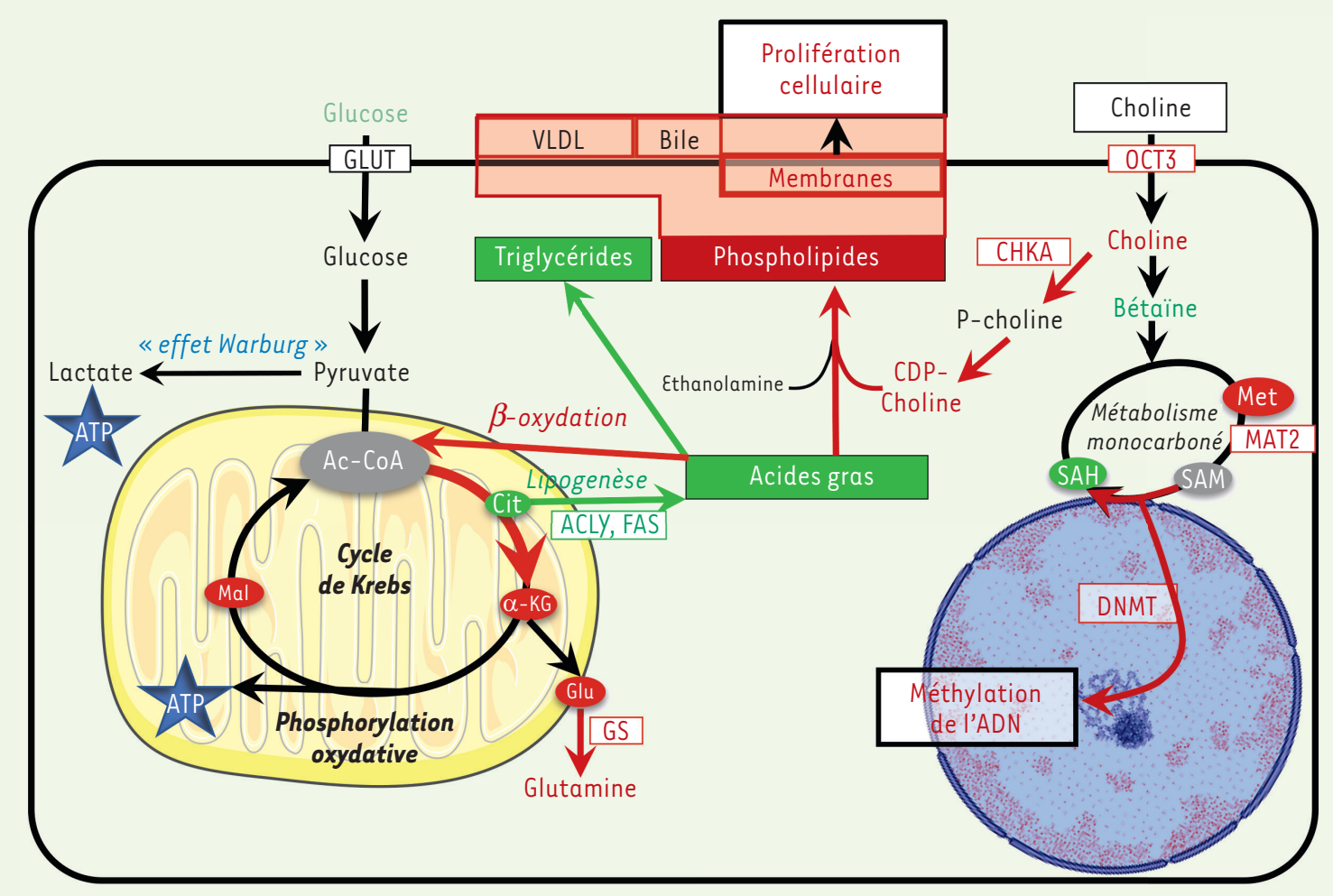

Figure 1. Métabolisme de l'hépatocyte présentant une activation de la signalisation $\beta$-caténine. L'augmentation de la quantité des métabolites et des ARN messagers des enzymes clés des voies métaboliques concernées par l'activation de la signalisation $\beta$-caténine est indiquée en rouge, tandis qu'une diminution est indiquée en vert. La glycolyse (à gauche sur la figure) n'est pas réorientée vers la production de lactate (effet Warburg), mais vers une production accrue de glutamine. La lipogenèse est diminuée, alors que la $\beta$-oxydation des acides gras constitue la source énergétique principale. Enfin, une captation accrue de choline alimentaire par le transporteur OCT3 permet: (1) la synthèse accrue de phospholipides, constituant de la bile, des VLDL, et surtout des membranes cellulaires, favorisant ainsi la prolifération cellulaire; (2) son oxydation en bétaïne et son rôle de pourvoyeur de groupements méthyle, en favorisant le métabolisme monocarboné et la méthylation de l'ADN. OCT3, organic cation transporter 3 ; CHKA, choline kinase $\alpha$; CDP-choline, cytidine-diphosphate-choline ; Met, méthionine; SAM/SAH, S-adénosylméthionine/S-adénosylhomocystéine ; MAT2, méthionine adénosyltransférase 2 ; DNMT, DNA methyltransferase ; GLUT, glucose transporter ; Cit, citrate ; $\alpha$-KG, $\alpha$-kétoglutarate ; Glu, glutamate ; ACLY, ATP citrate lyase ; FAS, fatty acid synthase.

génétiques ont été identifiées, et un tiers d'entre elles activent la voie de signalisation Wnt/ $\beta$-caténine. Cette voie, essentielle pour le développement embryonnaire et l'homéostasie des cellules souches adultes, joue un rôle physiologique particulier dans le foie. Elle y contrôle la régionalisation de ses fonctions métaboliques en contrôlant la transcription des enzymes et des transporteurs qui les assurent. Dans le CHC, la TEP utilisant le FDG (TEP-FDG) n'est pas la méthode de choix, puisqu'environ $30 \%$ des CHC ne présentent pas d'accroissement d'importation du FDG. Aussi, les tumeurs du foie sont diagnostiquées en première intention par échographie, dont la sensibilité (<80\%) n'est cependant pas optimale. Une imagerie par tomodensitométrie aux rayons $X$ (scanner) ou par résonance magnétique nucléaire (IRM) est souvent requise pour confirmer le diagnostic évoqué par l'échographie. Ces examens permettent aussi de repérer des nodules additionnels et d'éventuelles extensions de la tumeur à d'autres organes (ganglions lymphatiques, os, poumon, glande surrénale, péritoine). Plus récemment, l'imagerie morphologique a été complétée par des techniques d'imagerie fonctionnelle: IRM de diffusion, IRM ou scanner avec agent de contraste hépato-spécifique, et enfin TEP, avec ses limitations.
Dans le CHC, le glucose n'est pas le seul substrat énergétique utilisé par les celIules tumorales, qui peuvent également utiliser les acides gras, la glutamine, ou la choline [3, 4] (Figure I). C'est pour cette raison qu'une TEP utilisant la ${ }^{18} \mathrm{~F}$-choline (TEP-FCh), qui permet une visualisation des tumeurs les plus différenciées, a été proposée par nos collaborateurs comme alternative à la TEP-FDG dès 2010 [5] (Figure 2). La choline, initialement classée dans la famille des vitamines $B$, est un nutriment essentiel apporté principalement par l'alimentation et capté de manière active par le foie. Elle entre dans la composition de certains phospholipides 


\section{CHC avec mutation activatrice de CTNNB1 et TEP $-{ }^{18} \mathrm{FCh}$ « positif »}

Valeur standardisée de capture de la FCh $=23$
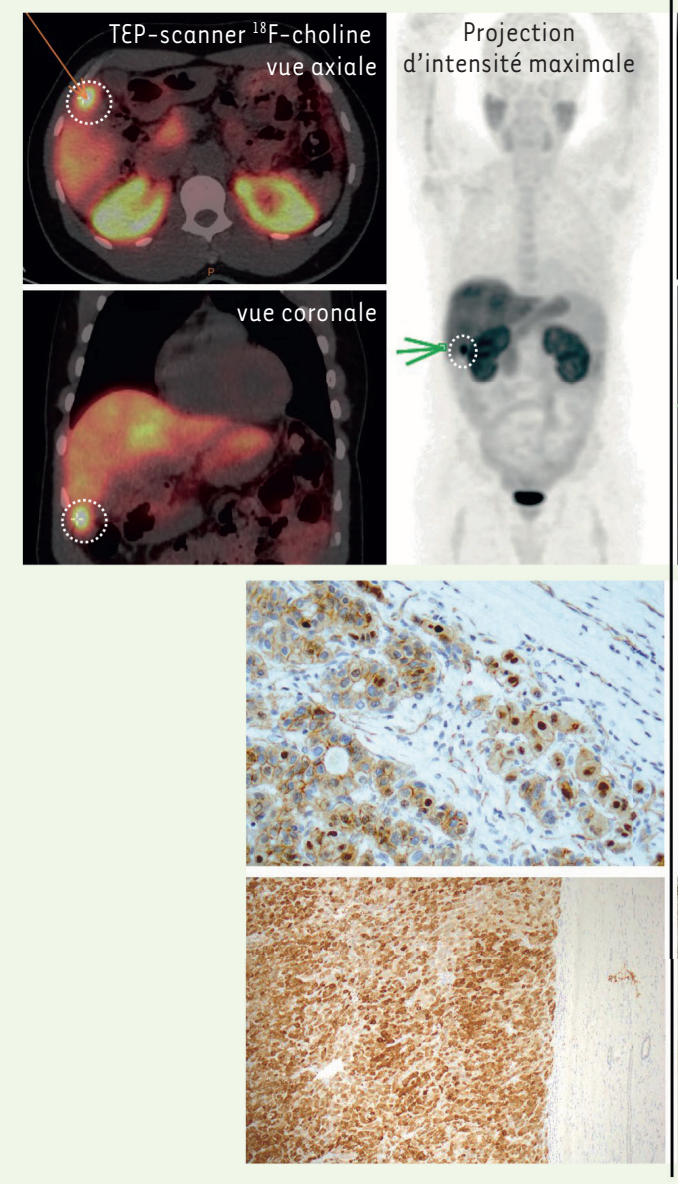

\section{CHC sans mutation de CTNNB1}

et TEP- ${ }^{18} \mathrm{FCh}$ 《 négatif 》

Valeur standardisée de capture de la FCh = 6
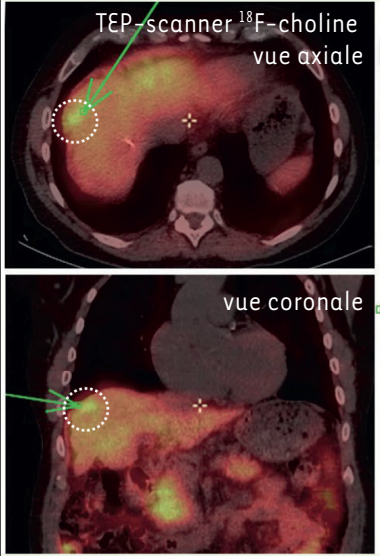

Projection

d'intensité maximale
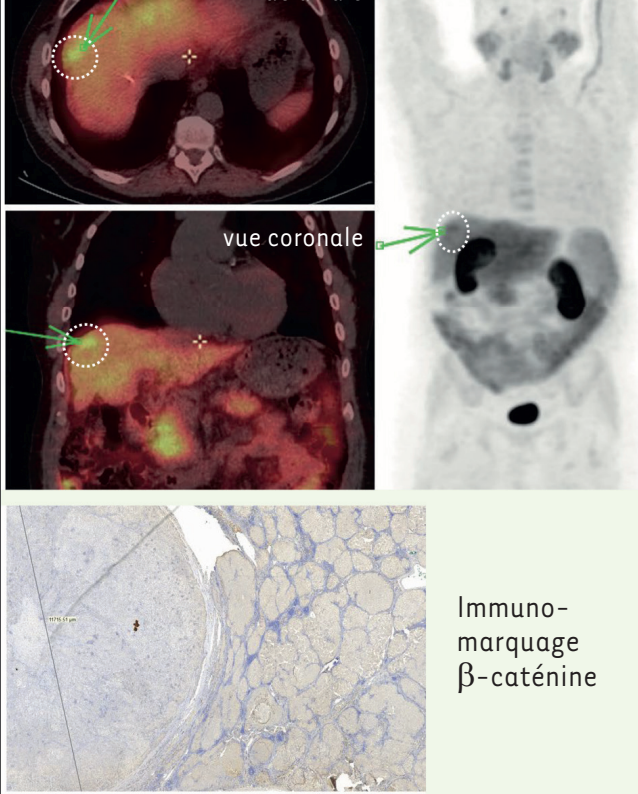

Immuno-

marquage

$\beta$-caténine

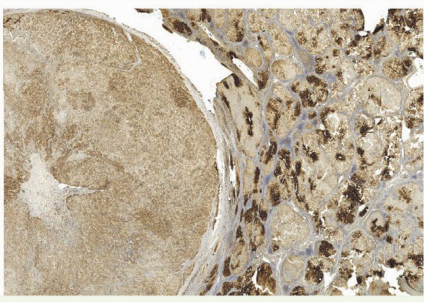

Immuno-

marquage GS

Figure 2. Tomodensitométrie associée à une TEP 18F-choline chez deux patients porteurs de CHC. Le premier CHC (à gauche), qui présente une captation accrue de ${ }^{18} \mathrm{FCh}$ mesurée par sa SUV (standardized uptake value) (valeur de capture standardisée) maximale, a été caractérisé par immunohistochimie pour son statut quant à l'expression de la $\beta$-caténine. La détection de la $\beta$-caténine dans le cytoplasme et le noyau des cellules tumorales, mais pas dans les cellules du foie non tumoral adjacent, indique l'activation de la voie de signalisation Wnt/ $\beta$-caténine dans les cellules tumorales. Cette activation est confirmée par l'abondance, dans ces cellules, de la glutamine synthétase (GS), qui est une cible transcriptionnelle de la $\beta$-caténine, permettant le diagnostic in situ de cette classe de tumeur. A l'inverse, le deuxième CHC (à droite) ne présente ni captation accrue de la ${ }^{18} \mathrm{FCh}$, ni activation de la voie $\beta$-caténine. La détection de la glutamine synthétase dans le foie non-tumoral adjacent correspond aux zones péricentrales, dans lesquelles il existe une activation physiologique de la signalisation $\beta$-caténine (figure adaptée de [9]).

des membranes cellulaires et est, par ailleurs, à l'origine de la voie de la bétaïne, pourvoyeur de groupements méthyle pour la méthylation de l'ADN notamment. Dans le foie, les phospholipides sont également un constituant majeur des VLDL (very low density lipoproteins) permettant l'exportation des lipides et de la bile, participant ainsi à la sécrétion des acides biliaires.
Addiction à la choline du CHC présentant des mutations activatrices du gène de la $\beta$-caténine

Notre équipe s'est intéressée à l'addiction à la choline d'un sous-groupe de CHC dont les cellules tumorales sont porteuses d'une mutation du gène de la $\beta$-caténine (CTNNBI) induisant une activation aberrante de cette voie de signalisation. Par un crible «métabolomique » et un séquençage des ARN et des produits d'immunoprécipitation de la chromatine obtenus avec un anticorps anti- $\beta$-caténine, réalisés dans un modèle murin préclinique mimant ce sous-type de CHC (Apc $\left.{ }^{\Delta H e p}\right)$ [6-8], nous avons observé une induction massive du métabolisme de la choline associée à 
une accumulation de phospholipides et une captation accrue de choline alimentaire [9] (Figure 1). Des études de flux réalisées sur des hépatocytes pré-tumoraux et sur des tumeurs isolées à partir de ces souris modèles ont confirmé que la captation accrue de choline radiomarquée par les hépatocytes $A p c^{\Delta H e p}$ entraîne une synthèse exacerbée de phospholipides contenant de la choline, et fournit des groupements méthyle contribuant à l'hyperméthylation de I'ADN tumoral. L'addiction à la choline regroupe ainsi les caractéristiques principales des cellules cancéreuses hépatiques présentant une hyperactivation de la voie $\beta$-caténine: hyperméthylation de I'ADN, prolifération, et absence de stéatose.

Cette captation accrue de la choline permet également une imagerie du carcinome hépatocellulaire par TEP-FCh chez l'homme (Figure 2). Nous avons analysé de manière rétrospective deux groupes de patients atteints de $\mathrm{CHC}$, chez lesquels une double imagerie de la tumeur par TEP-FDG et TEP-FCh avait été réalisée, et avons annoté le statut mutationnel des 49 tumeurs analysées. La fixation accrue de $\mathrm{FCh}$ par les $\mathrm{CHC}$ présentant une mutation de CTNNBI a permis de discriminer, par TEP-FCh, ces tumeurs de celles dépourvues d'une telle mutation, majoritairement indétectables avec cette technique, alors que la TEP-FDG n'était pas discriminante. Nous avons ainsi démontré la valeur de la TEP-FCh pour prédire un oncogénotype «CTNNBI-muté » dans le CHC. Les résultats de notre étude confirment et complètent d'autres résultats publiés la même année, révélant l'association entre détection par TEP-FCh, transcriptome tumoral, et survie des patients [10]. Dans cette étude, l'avidité des CHC pour la FCh était associée à un pronostic favorable de tumeurs caractérisées par la présence de mutations dans des gènes impliqués dans la phosphorylation oxydative et dans le métabolisme des acides gras, des acides biliaires, des xénobiotiques, et du peroxysome. En ce sens, nous avons également montré que l'oxydation des acides gras était fortement augmentée dans le foie des souris $A p c^{\Delta H e p}$, ce qui contribue à l'absence de stéatose hépatique observée chez ces souris et dans ce sous-type de $\mathrm{CHC}$ chez les patients. De plus, en l'absence du récepteur activé par les proliférateurs de peroxysomes (peroxisome proliferator-activated receptor $\alpha$, PPAR $\alpha$ ), la progression des tumeurs des souris $A p c^{\Delta H e p}$ est réduite, démontrant ainsi la dépendance énergétique aux acides gras des CHC mutés dans CTNNBI [3]. Ce sous-type de CHC est un exemple de tumeurs qui ne suivent pas le modèle historique de Warburg.

Notre étude a apporté la première démonstration en cancérologie qu'une imagerie peu invasive de la tumeur peut prédire son génotype et fournir un outil diagnostique informatif de l'évènement mutationnel à l'origine du développement tumoral. Elle est complémentaire d'une étude similaire portant sur le gliome, qui a montré une imagerie TEP-FCh différentielle des gliomes présentant une délétion $1 p / 19 q$ ou une mutation du gène codant l'isocitrate déshydrogénase [11]. Ainsi, I'utilisation de différents traceurs pour la TEP permettrait d'élargir le champ des possibilités diagnostiques pour de nombreux cancers (prostate, poumon, foie, etc.).

\section{Réduire l'apport alimentaire en} choline : une piste thérapeutique pour le $\mathrm{CHC}$ présentant une mutation activatrice du gène de la $\beta$-caténine ? Au-delà de la mise en évidence de l'intérêt diagnostique de la TEP-FCh, notre étude montre également que l'absence de choline dans l'alimentation modifie la reprogrammation épigénétique des hépatocytes $A p c^{\Delta H e p}$ précancéreux en réduisant le niveau de méthylation de leur ADN, limitant ainsi la prolifération de ces cellules à des stades précoces du processus tumorigénique [9]. De plus, la privation en choline alimentaire freine la progression des tumeurs
$A p c^{\Delta H e p}$ constituées, ce qui ouvre la voie à une stratégie d'intervention thérapeutique au carrefour du métabolisme et de l'épigénétique. Chez les patients porteurs de $\mathrm{CHC}$ présentant une mutation activatrice de CTNNB1, il est difficile d'envisager une restriction sévère prolongée en choline alimentaire étant donné son caractère essentiel, mais l'effet d'un régime alimentaire appauvri en choline sur la progression de la tumeur mériterait cependant d'être étudié. Par ailleurs, l'utilisation d'inhibiteurs pharmacologiques de la choline kinase $\alpha$ (CHKA), qui phosphoryle la choline au cours de la première étape de la synthèse des phospholipides, fait actuellement l'objet d'essais cliniques de Phase I en cancérologie, en sachant que l'utilisation thérapeutique de tels inhibiteurs ne pourrait pas empêcher les effets de la choline sur la méthylation de I'ADN des cellules tumorales. $\diamond$ Metabolic imaging assessing choline addiction in liver primary cancers predicts their oncogenotype and opens a new therapeutic avenue

\section{LIENS D'INTÉRÊT}

Les auteures déclarent n'avoir aucun lien d'intérêt concernant les données publiées dans cet article.

\section{RÉFÉRENCES}

1. Coatrieux JL, Velut J, Dillenseger JL, et al. De l'imagerie médicale à la thérapie guidée par l'image. Med Sci (Paris) $2010 ; 26: 1103-9$.

2. Cordier-Bussat M, Thibert C, Sujobert P, et al. Même l'effet Warburg est oxydable : coopération métabolique et développement tumoral. Med Sci (Paris) $2018 ; 34: 701-8$

3. Senni N, Savall M, Cabrerizo Granados D, et al. $\beta$-catenin-activated hepatocellular carcinomas are addicted to fatty acids. Gut $2019 ; 68: 322-34$.

4. Yuneva MO, Fan TW, Allen TD, et al. The metabolic profile of tumors depends on both the responsible genetic lesion and tissue type. Cell Metab $2012 ; 15$ : 157-70.

5. Talbot JN, Fartoux L, Balogova S, et al. Detection of hepatocellular carcinoma with $\mathrm{PET} / \mathrm{CT}$ : a prospective comparison of $18 \mathrm{~F}$-fluorocholine and $18 \mathrm{~F}-\mathrm{FDG}$ in patients with cirrhosis or chronic liver disease. J Nucl Med $2010 ; 51: 1699-706$.

6. Colnot S, Decaens T, Niwa-Kawakita M, et al. Liver-targeted disruption of Apc in mice activates $\beta$-catenin signaling and leads to hepatocellular carcinomas. Proc Natl Acad Sci USA 2004 ; 101 : 17216-21.

7. Gougelet A, Sartor C, Bachelot L, et al. Antitumour activity of an inhibitor of miR-34a in liver cancer with $\beta$-catenin-mutations. Gut 2016 ; 65 : 1024-34. 


\section{RÉFÉRENCES}

8. Gougelet A, Torre C, Veber P, et al. T-cell factor 4 and $\beta$-catenin chromatin occupancies pattern zonal liver metabolism in mice. Hepatology 2014 ; 59 : 2344-57.

9. Gougelet A, Sartor C, Senni N, et al. Hepatocellular carcinomas with mutational activation of $\beta$-catenin require choline and can be detected by positron emission tomography. Gastroenterology 2019 ; 157 : 807-22.

10. Kwee SA, Tiirikainen M, Sato MM, et al.

Transcriptomics associates molecular features with

${ }^{18} \mathrm{~F}$-fluorocholine PET/CT imaging phenotype and its potential relationship to survival in hepatocellular carcinoma. Cancer Res 2019 ; 79 : 1696-704.
11. Garcia Vicente AM, Perez-Beteta J, Amo-Salas M, et al. ${ }^{18} \mathrm{~F}$-Fluorocholine PET/CT in the prediction of molecular subtypes and prognosis for gliomas. Clin Nucl Med 2019; 44 : e548-58. 